\title{
Effect of Rubus idaeus Extracts in Murine Chondrocytes and Explants
}

\author{
Morgane Bourmaud $^{1}$, Mylene Zarka ${ }^{1}$, , Romain Le Cozannet ${ }^{2}$, Pascale Fança-Berthon ${ }^{2}$, Eric Hay ${ }^{1}$

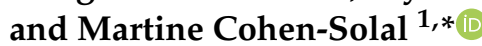 \\ 1 BIOSCAR Inserm U1132, Department of Rheumatology, Université de Paris, Hôpital Lariboisière, \\ F-75010 Paris, France; morgane.bourmaud@inserm.fr (M.B.); mylene.zarka@inserm.fr (M.Z.); \\ eric.hay@inserm.fr (E.H.) \\ 2 Naturex, Part of Givaudan SA, 250 rue Pierre Bayle, 84000 Avignon, France; \\ romain.le_cozannet@givaudan.com (R.L.C.); pascale.fanca-berthon@givaudan.com (P.F.-B.) \\ * Correspondence: martine.cohen-solal@inserm.fr; Tel.: +33-149-956-358
}

check for updates

Citation: Bourmaud, M.; Zarka, M.; Le Cozannet, R.; Fança-Berthon, P.; Hay, E.; Cohen-Solal, M. Effect of Rubus idaeus Extracts in Murine Chondrocytes and Explants. Biomolecules 2021, 11, 245. https:// doi.org/10.3390/biom11020245

Academic Editors: Monika Herten and Vladimir N. Uversky

Received: 27 October 2020

Accepted: 4 February 2021

Published: 9 February 2021

Publisher's Note: MDPI stays neutral with regard to jurisdictional claims in published maps and institutional affiliations.

Copyright: (C) 2021 by the authors. Licensee MDPI, Basel, Switzerland. This article is an open access article distributed under the terms and conditions of the Creative Commons Attribution (CC BY) license (https:/ / creativecommons.org/licenses/by/ $4.0 /)$.

\begin{abstract}
Osteoarthritis is characterized by cartilage loss resulting from the activation of chondrocytes associated with a synovial inflammation. Activated chondrocytes promote an increased secretion of matrix proteases and proinflammatory cytokines leading to cartilage breakdown. Since natural products possess anti-inflammatory properties, we investigated the direct effect of Rubus idaeus extracts (RIE) in chondrocyte metabolism and cartilage loss. The effect of RIE in chondrocyte metabolism was analyzed in murine primary chondrocytes and cartilage explants. We also assessed the contribution of RIE in an inflammation environment by culturing mice primary chondrocytes with the supernatant of Raw 264.7 macrophage-like cells primed with RIE. In primary chondrocytes, RIE diminished chondrocyte hypertrophy (Col10), while increasing the expression of catabolic genes (Mmp-3, Mmp-13) and reducing anabolic genes (Col2a1, Acan). In cartilage explants, Rubus idaeus prevented the loss of proteoglycan $(14.84 \pm 3.07 \%$ loss of proteoglycans with IL1 alone vs. $3.03 \pm 1.86 \%$ with IL1 and $100 \mu \mathrm{g} / \mathrm{mL}$ of RIE), as well as the NITEGE neoepitope expression. RIE alone reduced the expression of $I l 1$ and Il6 in macrophages, without changes in Tnf and Cox2 expression. The secretome of macrophages pre-treated with RIE and transferred to chondrocytes decreases the gene and protein expression of Mmp-3 and Cox2. In conclusion, these data suggest that RIE may protect from chondrocyte catabolism and cartilage loss in inflammatory conditions. Further evaluations are need before considering RIE as a candidate for the treatment for osteoarthritis.
\end{abstract}

Keywords: Rubus idaeus; chondrocytes; macrophages; osteoarthritis; inflammation

\section{Introduction}

Osteoarthritis (OA) is the most common cause of arthritis affecting millions of people worldwide. OA affects $30 \%$ of people over 65 years and is one of the most expensive chronic diseases in developed countries. Because of its function-impairing nature, the burden is high in terms of prevalence and economic impact [1,2]. Pain and dysmobility are major drivers of clinical decision making and health service use. In contrast to this high prevalence, there is no treatment available to slow or treat the loss of cartilage. The pathophysiology is underpinned by several factors that combine mechanical overload, genetic, and hormonal factors. Several clinical risk factors are identified such as age, obesity, and trauma, whilst others are not modifiable. The breakdown of the cartilage matrix that characterizes OA generates pain and functional disability, a source of high morbidity. Chondrocytes, the unique specialized cells in the cartilage, ensure the renewal of the extracellular matrix which keeps the metabolic properties of the cartilage and its mechanical skills. In $\mathrm{OA}$, chondrocytes form clusters and their activation results in increased secretion of matrix proteases (MMP-3, MMP-13, ADAMTS-5) capable of degrading the aggrecan and cleaving type II collagen network. Cartilage loss is also associated with a reduction in 
chondrocyte maintenance, a process that drives the commitment of chondrocytes into a hypertrophic maturation close to that observed during endochondral ossification. In addition to altered chondrocyte function, a synovial inflammation participates in cartilage loss through the invasion of immune cell infiltration that produces inflammatory cytokines that further activate chondrocyte catabolism [3]. Macrophages are key cellular mediators of innate immunity in OA [4] through their capacity to activate cytokines and MMPs by chondrocytes, but also by inhibiting the gene expression of anabolic genes such as COL2 and ACAN [5]. Macrophage secretome inhibits the proliferation and viability of chondrocyte stem cells, thereby modulating cartilage repair [6]. Several subpopulations, including polarized macrophages that regulate cartilage remodeling, have been identified and might be specific drug targets for OA.

Despite major advances in knowledge in disease mechanisms, $\mathrm{OA}$ is orphaned of structural treatment likely related to the diversity of tissues to target and the heterogeneity in clinical profile of OA patients. This leaves a place for natural products derived from herbs and plants that have biological effects and may prevent cartilage loss and joint pain. A large variety of compounds have been tested in cells or animal models [7], providing evidence of a potential use of natural products as an alternative or complementary treatment. These compounds act through different signaling pathways (NF- $\kappa \mathrm{B}$, apoptosis, MAP kinase) and target several genes such as inflammatory cytokines and MMPs. In addition, glucosamine or chondroitin possess anti-inflammatory and antioxidant properties that may inhibit the release of OA-related cytokines and slow down the progression of OA [8]. For example, glucosamine inhibits NF- $\kappa \mathrm{B}$ signaling in co-culture models of synoviocytes and chondrocytes [9], thereby reducing pain and functional disability [10,11].

Rubus idaeus, derived from raspberry fruits, contain flavonoids and phenols [12] that inhibit inflammatory processes. Other Rubus compounds such as Rubus fruticosus prevent the activation of MAPK or NFkB signaling in fibroblasts, thereby preserving the type-1 collagen against UVB [13]. Blackberry extracts reverse pathological processes in mice such as manic episodes [14], whilst Rubus idaeus extracts (RIE) prevent hyperlipidemia [15] and kidney stone formation [16]. Moreover, polyphenolic-enriched red raspberry extract reduces collagen breakdown in bovine chondrocytes as well as the severity of arthritis in an antigen-induced arthritis rat model [17]. Given the potential protection in cartilage loss, we investigated here the effect of RIE in cartilage and the contribution of inflammatory joint cells in chondrocyte catabolism.

\section{Materials and Methods}

\subsection{Rubus idaeus Extracts}

The hydroalcoholic (ethanol 30\%/water 70\%) Rubus idaeus aerial part was extracted from a native extract ratio of 3-5/1. The extract has been standardized to contain more than $10 \%$ of polyphenols with more than $0.4 \%$ of sanguiin H6. Briefly, sample preparation corresponded to approximately $400 \mathrm{mg}$ of extract of Rubus idaeus into a $250 \mathrm{~mL}$ volumetric flask, diluted to volume with methanol, followed by $10 \mathrm{~min}$ of sonication and at least filtering through a PTFE $0.45 \mu \mathrm{m}$ filter. Quantification of the target compound was performed on an HPLC Agilent 1100 HPLC system equipped with a UV detector. The separation of compounds was carried out on Atlantis T3 C18 $150 \times 3.0 \mathrm{~mm} ; 3 \mu \mathrm{m}$ set at $32{ }^{\circ} \mathrm{C}$. The mobile phase consisted of acetonitrile/water (50:50) $+0.1 \%$ formic acid (eluent A) and water $+0.1 \%$ formic acid (eluent B). The gradient for eluent $\mathrm{A}$ was as follows: $0 \mathrm{~min}, 12 \%$; $10 \mathrm{~min}, 20 \%$; $30 \mathrm{~min}, 43 \%$; $40 \mathrm{~min}, 100 \%$; $55 \mathrm{~min}, 100 \%$. The gradient for eluent $\mathrm{B}$ was the difference between $10 \%$ and eluent A gradient. The total run time was $50 \mathrm{~min}$, injection volume was $2 \mu \mathrm{L}$, and flow rate was $0.6 \mathrm{~mL} / \mathrm{min}$. UV monitoring was performed at $280 \mathrm{~nm}$ for sanguiin $\mathrm{H} 6$ compound detection. The amount of target compound was quantified by comparing the peak area of the sample with the peak area of the reference compound of known concentration. The total phenolic content was determined by using the Folin-Ciocalteu assay as previously described [18]. Colorimetry of total phenolics was performed with phosphomolybdic-phosphotungstic acid reagents. The extract powder 
was then diluted in PBS for cell and organ cultures just before each experiment; PBS alone was used as the control.

\subsection{Primary Chondrocyte Culture}

Primary articular chondrocytes were isolated from femoral heads, femoral condyles, and tibial plateaus of newborn C57BL/6J mice. Chondrocytes were isolated and cultured as described previously [19]. After incubation with liberase (Roche Applied Science, Penzberg, Germany) for $24 \mathrm{~h}$, chondrocytes were seeded at a density of 200,000 cells/mL in 12-well plates and were expanded in Dulbecco's Modified Eagle Medium (DMEM, Fisher Scientific, Hampton, New Hampshire, NH, USA) with 10\% decomplemented Fetal Calf Serum (FCS), 2\% L-glutamine, and 1\% penicillin/streptomycin until confluence. To see a direct effect of RIE on chondrocytes metabolism, chondrocytes were cultured for $24 \mathrm{~h}$ without FCS, then confluent monolayers were stimulated with $1 \mathrm{ng} / \mathrm{mL}$ of IL1 (R\&D Systems, Minneapolis, Minnesota, MO, USA) for $24 \mathrm{~h}$, washed, and cultured for an additional $24 \mathrm{~h}$ in the presence of increasing doses of 10,20,40, or $100 \mu \mathrm{g} / \mathrm{mL}$ of RIE. To see an effect in an inflammation environment, chondrocytes were cultured for $24 \mathrm{~h}$ with macrophage secretome primed with RIE.

At the end of the culture, cells were lysed, and then protein and RNA were extracted for further analysis. All experiments were performed at least three times.

\subsection{Macrophage Culture}

The raw 264.7 macrophage-like cells were cultured in DMEM supplemented with $10 \%$ of decomplemented FCS, $2 \%$ L-glutamine and $1 \%$ penicillin/streptomycin. Cells were seeded at a density of 100,000 cells/well in 6-well plates and incubated for $24 \mathrm{~h}$ at $37^{\circ} \mathrm{C}$ and $5 \% \mathrm{CO}_{2}$. Media of each well were collected and replaced with fresh media containing RIE at 10 or $20 \mu \mathrm{g} / \mathrm{mL}$. After $24 \mathrm{~h}$, cells were washed and stimulated with $100 \mathrm{ng} / \mathrm{mL}$ of lipopolysaccharides (LPS) for $2 \mathrm{~h}$. Then, cells were washed three times with PBS and cultured with fresh media for $24 \mathrm{~h}$. Supernatants were collected and stored at $-80{ }^{\circ} \mathrm{C}$. All experiments were performed three times and cells were used at the same passage for each new experiment.

\subsection{Cell Viability}

Cell viability was determined by non-specific redox enzyme activity using CellTiterBlue (Promega, Madison, Wisconsin, WI, USA). Macrophages were seeded in 96-well plates $(100 \mu \mathrm{L}, 3000$ cells/well) and cultured as described above. On the last day, $20 \mu \mathrm{L}$ of CellTiter-Blue Reagent was added to the wells and incubated for 1 hour at $37^{\circ} \mathrm{C}$ protected from light. Fluorescence was recorded and values of viability of treated cells were expressed as a percentage of corresponding control cells (excitation $560 \mathrm{~nm}$, emission at $590 \mathrm{~nm}$ ). All experiments were repeated three times in duplicate.

\subsection{Gene Expression}

RNA was isolated using TRIzol Reagent (Fisher Scientific, Hampton, New Hampshire, United States) and total RNA was reverse-transcribed with a High-Capacity cDNA Reverse Transcription Kit (Fisher Scientific, Hampton, New Hampshire, NH, United States). Relative mRNA levels were evaluated by quantitative Polymerase Chain Reaction (qPCR) using a SENSIFast SYBR No-ROX kit (Bio-Technofix, Guibeville, France) and were normalized to the level of Glyceraldehyde-3-Phosphate Dehydrogenase (Gapdh) for chondrocytes and with TATA-Box Binding Protein (Tbp) and Beta-Actin for macrophages. Relative expression was calculated by the comparative $\mathrm{Ct}$ method $(-\Delta \Delta \mathrm{Ct})$.

Genes expression of catabolic markers (Mmp-3, Mmp-13, Adamts-5), anabolic markers (Collagen type II, Aggrecan), hypertrophic markers (Collagen type X, Vegf), and inflammation (Il1, Il6, Cox2, Tnf) were evaluated. Primers sequences were as follows:

Mmp-3, 5'-AAAGACAGGCACTTTTGGCG-3' and 5'-CATGTTGGATGGAAGAGATGGC-3'; Mmp-13, 5' -TGATGGCACTGCTGACATCAT-3' and 5'-TGTAGCCTTTGGAACTGCTT-3'; 
Adamts-5, $5^{\prime}$-TCAGCCACCATCACAGAA- ${ }^{\prime}$ and $5^{\prime}$-CCAGGGCACACCGAGTA- ${ }^{\prime}$; Aggrecan, 5'-CAGGGTTCCCAGTGTTCAGT-3' and 5'-CTGCTCCCAGTCTCAACTCC-3'; Collagen type II (Col2a1), 5'-CCGTCATCGAGTACCGATCA-3' and 5'-CAGGTCAGGTCAGCCATTCA-3'; Collagen type X (Col10a1), 5'-AAGGAGTGCCTGGACACAAT-3' and 5'-GTCGTAATGCTGCTGCCTAT-3';

Vegf, $5^{\prime}$-GGAGATCCTTCGAGGAGCACTT- ${ }^{\prime}$ and $5^{\prime}$-GGCGATTTAGCAGCAGATATAAGAA- $3^{\prime}$;

Gapdh 5' $^{\prime}$-AACTTTGGCATTGTGGAAGG-3' and $5^{\prime}$-ACACATTGGGGGTAGGAACA- $3^{\prime}$; Il1, $5^{\prime}$-CCTCACAAGCAGAGCACAAG- $3^{\prime}$ and $5^{\prime}$-AAACAGTCCAGCCCATACTTTAG- $3^{\prime}$; Il6, 5' -CAAAGCCAGAGTCCTTCAGAG-3' and $5^{\prime}$-GGATGGTCTTGGTCCTTAGC-3'; Cox $2,5^{\prime}$ - CCAGCACTTCACCCATCAGTT- $3^{\prime}$ and $5^{\prime}$ - ACCCAGGTCCTCGCTTATGA- $3^{\prime}$; Tnf, $5^{\prime}$-CCCATGTTGTAGCAAACCCTC- $3^{\prime}$ and $5^{\prime}$-TATCTCTCAGCTCCACGCCA- $3^{\prime}$; Beta-Actin, 5'-GGCTGTATTCCCCTCCATCG-3' and 5'-CCAGTTGGTAACAATGCCATGT- ${ }^{\prime}$;

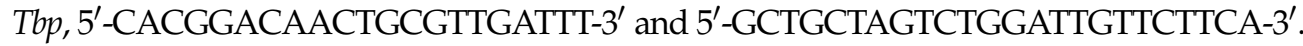

\subsection{Culture of Cartilage Explants}

To assess the effect of RIE in the joints, we used cartilage explants obtained from femoral heads of 10-week-old C57BL/6J mice as described [20]. Femoral head explants were cultured in FCS-free DMEM for $24 \mathrm{~h}$, and then stimulated for $72 \mathrm{~h}$ with $10 \mathrm{ng} / \mathrm{mL}$ of IL1 with or without 10 or $100 \mu \mathrm{g} / \mathrm{mL}$ of RIE. Explants were prepared for cryosection $(\mathrm{n}=3$ explants per condition).

\subsection{Histology and Immunohistochemistry}

Cartilage explants were fixed with $4 \%$ PFA for $24 \mathrm{~h}$ at $4{ }^{\circ} \mathrm{C}$, and then decalcified with $0.5 \mathrm{M}$ EDTA at room temperature for $24 \mathrm{~h}$, changing the solution twice. Frozen sections ( $5 \mu \mathrm{m}$ thick) of Optimal Cutting Temperature (OCT)-embedded cartilage explant were cut for histology and immunohistochemistry procedures. Cartilage breakdown was assessed on serial sections collected in 3 different zones stained with safranin-O. Sections were stained using Mayer's hemalum for 3 min to stain the nuclei, rinsed with water three times, and then counterstained with $0.125 \%$ Fast Green for $10 \mathrm{~min}$ to visualize bone tissue. After being rinsed in two successive $1 \%$ acetic acid baths, they were stained in $0.5 \%$ Safranin-O $10 \mathrm{~min}$, and then rinsed in $100 \%$ ethanol. To quantify the staining loss, which reveals a loss of sulfated proteoglycans, we measure the area with loss of staining, which appears in blue and the total area in red. Results are presented as the ratio of cartilage without proteoglycan staining over the total area.

Immunohistochemistry was performed on serial sections. Primary polyclonal antibodies directed against murine NITEGE neoepitope (1:100, Fisher Scientific, Hampton, NH, USA) were used to assess aggrecanase expression and localization. A DAB Peroxidase Substrate Kit (Vector Laboratories, Burlingame, CA, USA) was used for revelation and sections were counterstained with methyl green.

\subsection{Western Blotting}

The protein expression levels in the culture medium from femoral heads or protein lysate of chondrocytes were detected by Western blot. First, $10 \mu \mathrm{g}$ of protein lysate were resolved in $4-12 \%$ SDS-PAGE under reducing conditions and transferred to a PDVF membrane. The membrane was then blocked for nonspecific binding in casein for $1 \mathrm{~h}$ and incubated with primary antibody MMP-3 (1:500, ab52915, Abcam, Cambridge, UK), or COX2 (1:500, sc-1745, Santa Cruz Biotechnology, Dallas, TX, USA) overnight at $4{ }^{\circ} \mathrm{C}$. After being washed, membranes were incubated with peroxidase-conjugated anti-rabbit for $1 \mathrm{~h}$ at room temperature and then, developed using a blotting detection kit (Radiance Plus Revelation ECL, Azure Biosystems, Dublin, OH, USA). 


\subsection{Statistical Analysis}

To determine the statistical significance, analysis was conducted by ANOVA and then by a Mann-Whitney or Kruskal-Wallis test. $p<0.05$ was considered statistically significant. Data are expressed as mean \pm SEM from at least three experiments.

\section{Results}

\subsection{Dose-Effect on Cell Viability}

The dose-effect of RIE was assessed to test the cell viability and to choose the dose for subsequent experiments. At the dose of 40 and $100 \mu \mathrm{g} / \mathrm{mL}$, RIE promoted the death of primary chondrocytes after $48 \mathrm{~h}$ of culture. Subsequently, further experiments were conducted with the doses of 10 and $20 \mu \mathrm{g} / \mathrm{mL}$ that do not alter cell viability. Similar tests were conducted on macrophages. Ten and $20 \mu \mathrm{g} / \mathrm{mL}$ of RIE do not alter the cell viability of macrophages $(106.13 \pm 5.52 \%, 103.59 \pm 9.39 \%$, and $96.10 \pm 5.24 \%$ viable cells with LPS, 10 or $20 \mu \mathrm{g} / \mathrm{mL}$ RIE, respectively, compared to control cells) (Supplemental Figure S1).

\subsection{Effects of Rubus idaeus in the Expression of Catabolic and Anabolic Genes}

The effects of RIE were assessed by culturing primary murine chondrocytes (Figure 1). RIE alone significantly increased the expression of $M m p-3$ at 10 and $20 \mu \mathrm{g} / \mathrm{mL}(18.70 \pm 12.81$ and $136.96 \pm 109.06$-fold, respectively, $p<0.05$ ) and the expression of $M m p-13$ at $20 \mu \mathrm{g} / \mathrm{mL}$ ( $8.72 \pm 5.84$-fold, $p<0.05)$. In contrast, the highest dose of RIE was required to decrease the expression of Adamts -5 at $20 \mu \mathrm{g} / \mathrm{mL}$ compared to controls $(0.62 \pm 0.07$-fold, $p<0.05)$ (Figure 1A). We then tested the effect on chondrocytes primed with IL1 to mimic an inflammatory context. IL1 alone induced a significant increase in the expression of catabolic markers: $255.13 \pm 99.22$-fold change $(p<0.05)$ for $M m p-3,7.34 \pm 1.56$-fold change $(p<0.05)$ for Mmp-13. However, there was no effect of addition of RIE and IL1 compared to IL1 alone for the expression of Mmp-3, Mmp-13, and Adamts-5 at any dose.

Investigating the effects in anabolic genes compared to controls, IL1 alone reduced the expression of Col2a1 ( $0.31 \pm 0.11$-fold) and Acan ( $0.36 \pm 0.13$-fold), both $p<0.05$. Compared to controls, RIE alone reduced Col2a1 $(0.73 \pm 0.05$ and $0.43 \pm 0.22$-fold at 10 and $20 \mu \mathrm{g} / \mathrm{mL}$, respectively, $p<0.05)$ and Acan levels $(0.52 \pm 0.10$ and $0.24 \pm 0.09$-fold at 10 and $20 \mu \mathrm{g} / \mathrm{mL}$, respectively, $p<0.05$ ) (Figure 1B). However, the addition of RIE and IL1 has no significant effect compared to IL1 alone for the expression of Col2a1 and Acan. Hypertrophic markers revealed no significant changes in Col10a1 and Vegf expression at RIE concentration of $10 \mu \mathrm{g} / \mathrm{mL}(1.46 \pm 0.49$ and $0.88 \pm 0.10)$, but a reduction at $20 \mu \mathrm{g} / \mathrm{mL}(0.40 \pm 0.06$ and $0.78 \pm 0.10, p<0.05$ ) compared to controls (Figure 1C). Col10a1 expression was significantly lower in IL1-stimulated chondrocytes $(0.25 \pm 0.07, p<0.05)$, whilst the addition of IL1 and RIE further decreased in the two doses, without reaching significance. Lower doses of 1 and $5 \mu \mathrm{g} / \mathrm{mL}$ provided similar results.

\subsection{Effects of Rubus idaeus Extracts in Cartilage Loss and Proteinases in Cartilage Explants}

To test the effect of RIE for cartilage degradation, we used the model of murine cartilage explants. Explants were cultured alone or stimulated by IL1 with or without two different doses of RIE. Figure 2A shows that RIE alone has no effect compared to controls. However, RIE prevented the loss of proteoglycan assessed by Safranin O at the dose of $100 \mu \mathrm{g} / \mathrm{mL}$ in the presence of IL1 (3.03 $\pm 1.86 \%)$ compared to IL1 alone $(14.84 \pm 3.07 \%)$, although not statistically significantly. No effect was observed at $10 \mu \mathrm{g} / \mathrm{mL}$ of RIE $(16.21 \pm 1.21 \%$ staining loss in the presence of $10 \mu \mathrm{g} / \mathrm{mL}$ and IL1). Moreover, addition of RIE did not change the localization and expression of NITEGE neoepitope at $100 \mu \mathrm{g} / \mathrm{mL}$ in explants treated with IL1 $(66.05 \pm 5.87 \%$ positive cells with IL1 alone compared to $37.93 \pm 10.64 \%$ positive cells with IL1 and $100 \mu \mathrm{g} / \mathrm{mL}$ ) (Figure 2B). 

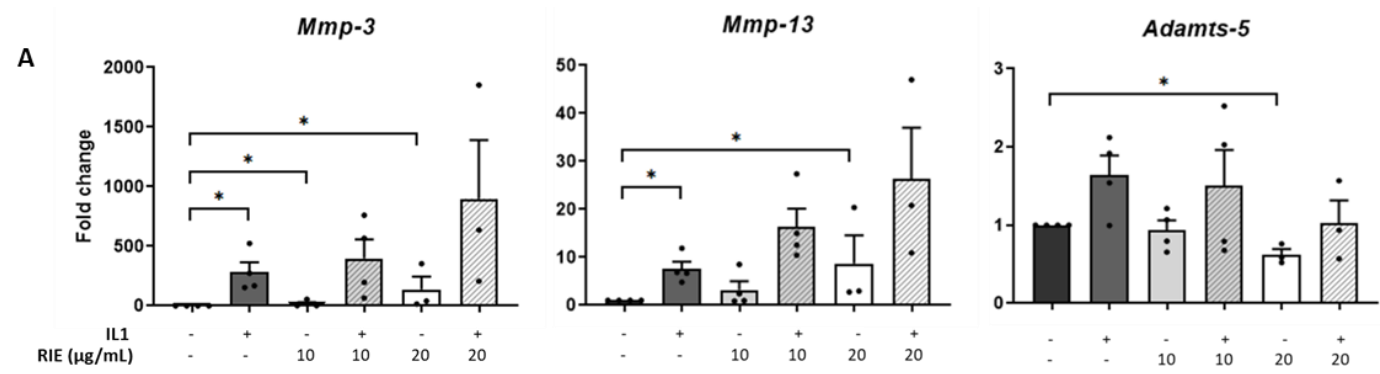

B
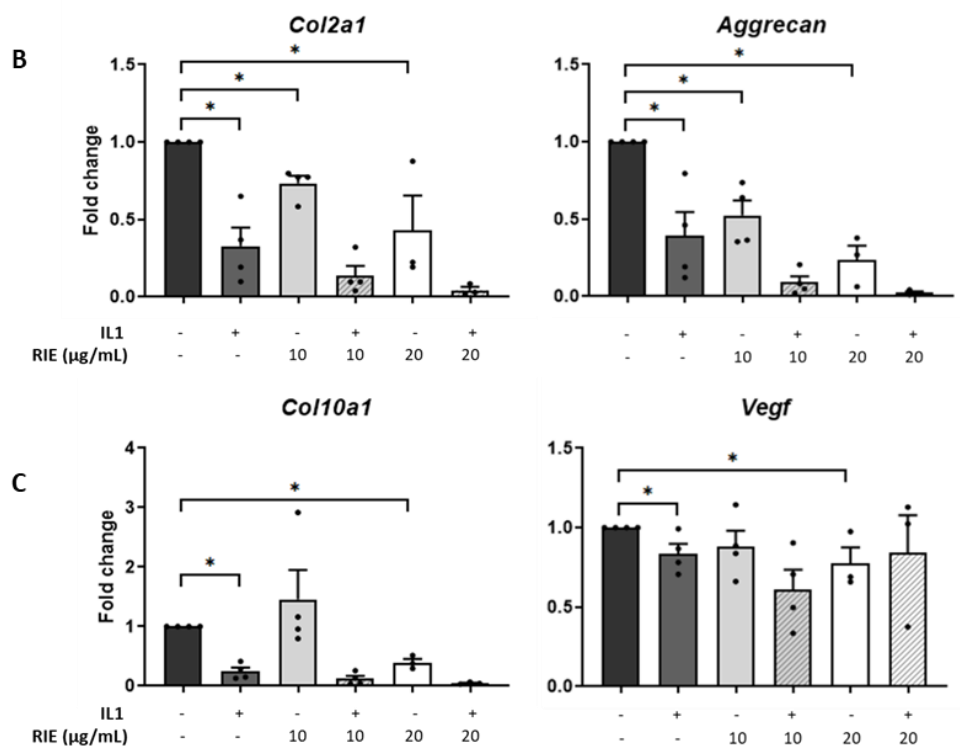

Figure 1. Effects of Rubus idaeus in catabolic, anabolic, and hypertrophic genes in primary murine chondrocytes. qPCR analysis of catabolic genes Mmp-3, Mmp-13, and Adamts-5 (A), anabolic genes Col2a1 and Acan (B), and hypertrophic genes Col10a1 and $\operatorname{Veg} f(\mathbf{C})$ in chondrocytes stimulated with IL1 and then treated $24 \mathrm{~h}$ with Rubus idaeus extracts (RIE) $(10 \mathrm{or} 20 \mu \mathrm{g} / \mathrm{mL})$. Data are mean \pm SEM from four experiments. ${ }^{*} p<0.05$.

A

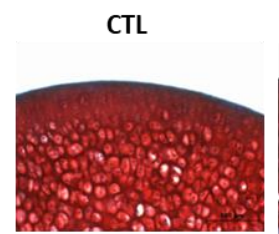

RIE $10 \mu \mathrm{g} / \mathrm{mL}$
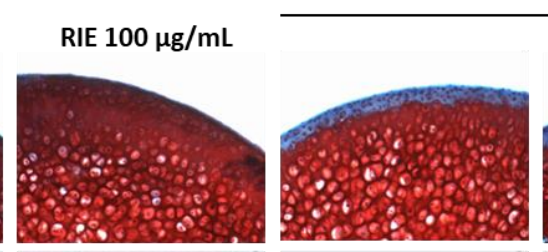

IL1
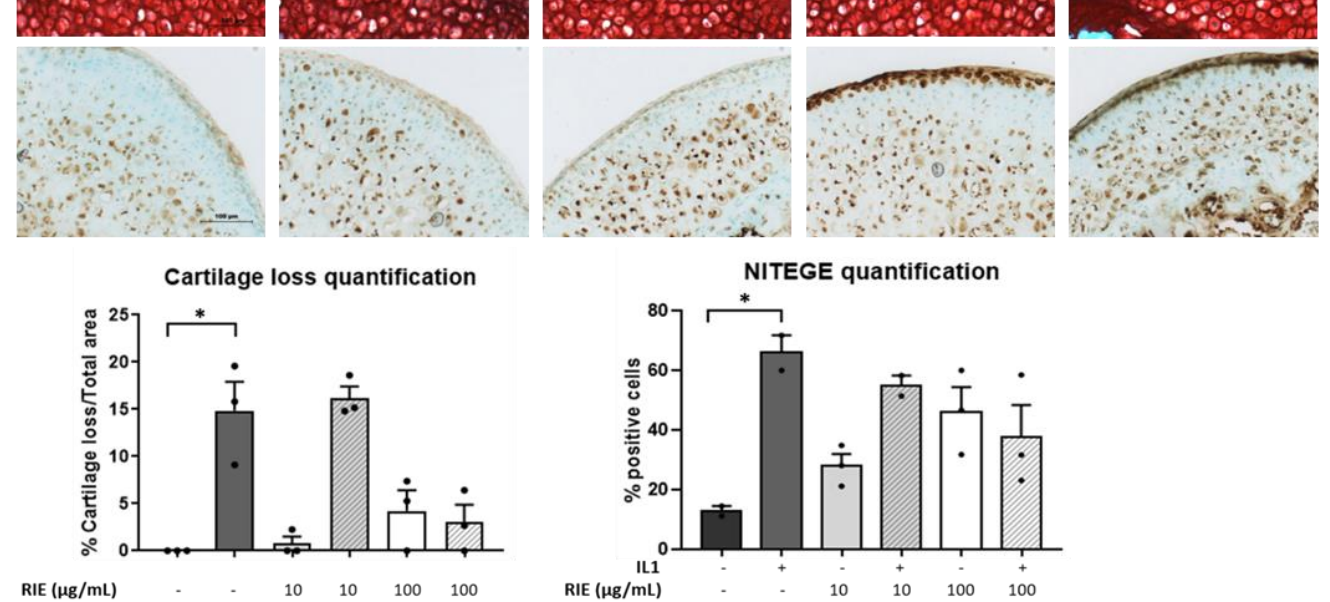

Figure 2. Effect of RIE in cartilage composition and NITEGE expression in murine cartilage explants. Safranin O staining, NITEGE neoepitope immunohistochemistry (A) and the associated quantification (B) of cryosections of mouse femoral head cartilage explants treated or not with IL1 and RIE $(10$ or $100 \mu \mathrm{g} / \mathrm{mL})$ for $72 \mathrm{~h} .{ }^{*} p<0.05$. 


\subsection{Effects of Rubus idaeus Extracts in Inflammatory Genes in Macrophages}

To test the role of RIE in inflammatory conditions, macrophages were pre-treated with two different doses of RIE, and then stimulated with LPS. RIE alone did not modify the expression of Il1, Il6, or Cox2, but slightly increased the Tnf expression at $20 \mu \mathrm{g} / \mathrm{mL}$ $(1.22 \pm 0.09$-fold change compared to controls, $p<0.05)$. Compared to control cells, LPS alone increased the expression of inflammatory genes (507.89 \pm 258.99 -fold for Il1, $5.97 \pm 1.55$-fold for $I l 6,18.41 \pm 2.95$-fold for Tnf, and $7.10 \pm 1.29$-fold for Cox $2, p<0.05$ ) (Figure 3). However, there was no change in the expression of any gene expression when comparing the addition of RIE and LPS to LPS alone.
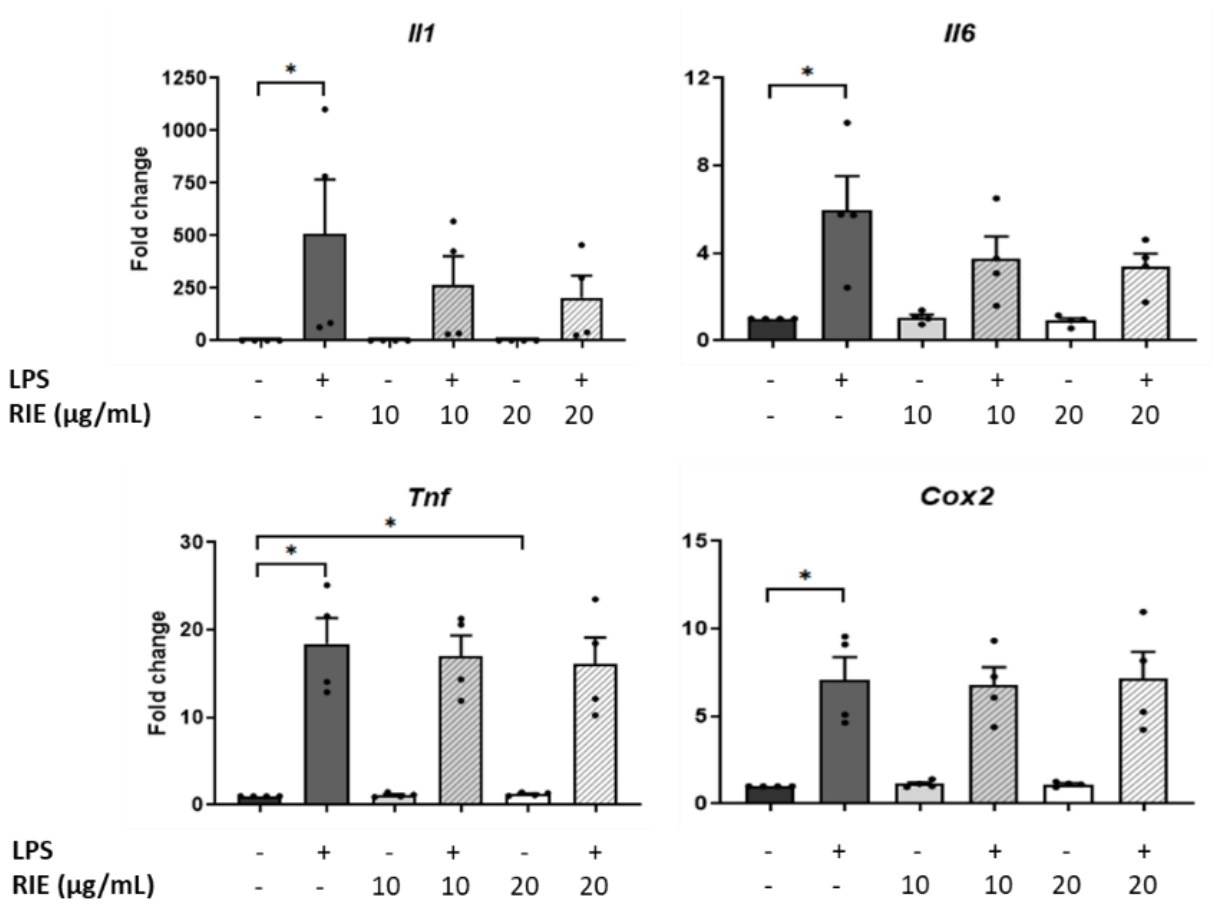

Figure 3. Expression of inflammatory cytokines in murine macrophages primed with Rubus idaeus. qPCR analysis of inflammatory genes, Il1, Il6, Tnf, and Cox2 in macrophages pre-treated with RIE at two different doses (10 or $20 \mu \mathrm{g} / \mathrm{mL})$ and then stimulated with LPS (100 $\mathrm{ng} / \mathrm{mL})$. Data are mean \pm SEM from four experiments. ${ }^{*} p<0.05$.

\subsection{Effects of Secretome of Macrophages Primed with RIE in Chondrocytes}

We then assessed the effect of the secretome of RIE-primed macrophages in primary murine chondrocytes. Secretome of macrophages primed with 10 or $20 \mu \mathrm{g} / \mathrm{mL}$ of RIE alone did not affect the expression of catabolic, anabolic, or inflammatory genes of chondrocytes in basal conditions compared to controls (Figure 4A,B). Therefore, macrophages were primed with RIE and then stimulated with LPS; the supernatant was transferred to the chondrocytes cultures. Compared to the controls, the secretome of LPS-primed macrophages stimulated the expression of catabolic genes (81.02 \pm 23.52 -fold for $\mathrm{Mmp}$-3, $11.31 \pm 1.56$-fold for Mmp-13, and $3.23 \pm 0.42$-fold for Adamts-5, $p<0.05$, Figure 4A). Compared to LPS alone, chondrocytes cultured with the secretome of RIE primed-macrophages and LPS revealed a trend toward reduced expression of Mmp-3, Mmp-13, and Adamts-5, but this reduction did not achieve statistical significance. 
Compared to controls, the expression of Cox 2 was activated upon LPS (4.61 \pm 0.94 -fold, $p<0.05)$. However, the secretome of RIE-primed macrophages did not significantly change the expression of Cox 2 compared to LPS alone (5.31 \pm 1.70 -fold and $2.60 \pm 0.47$-fold) (Figure 4B). Moreover, LPS significantly reduced the expression of anabolic gene Aggrecan $(0.43 \pm 0.08$-fold compared to control, $p<0.05)$, but no additional effect was observed with the secretome of RIE-primed macrophages. Neither LPS nor RIE affected the expression of the Col2a1 gene.
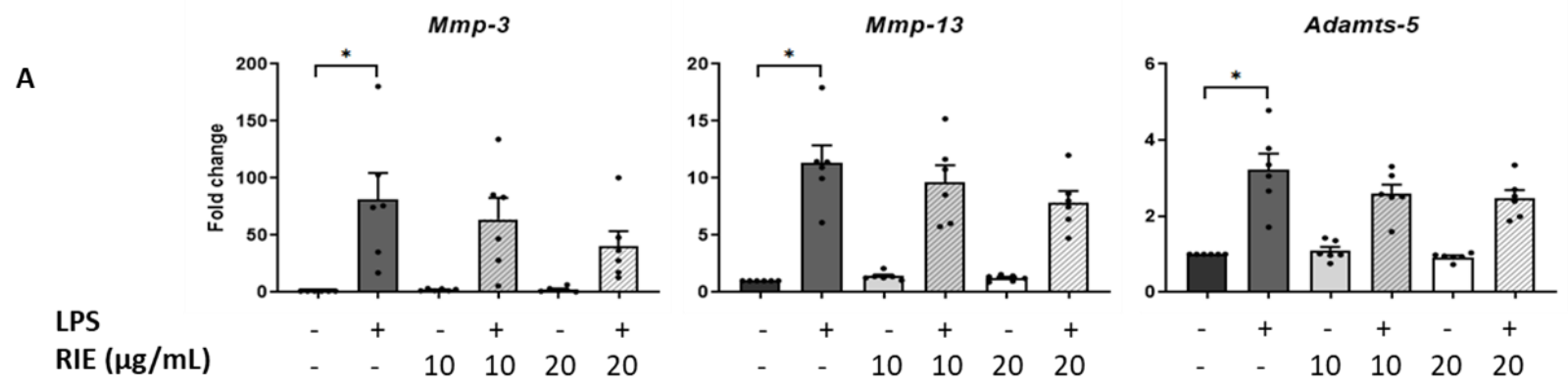

B

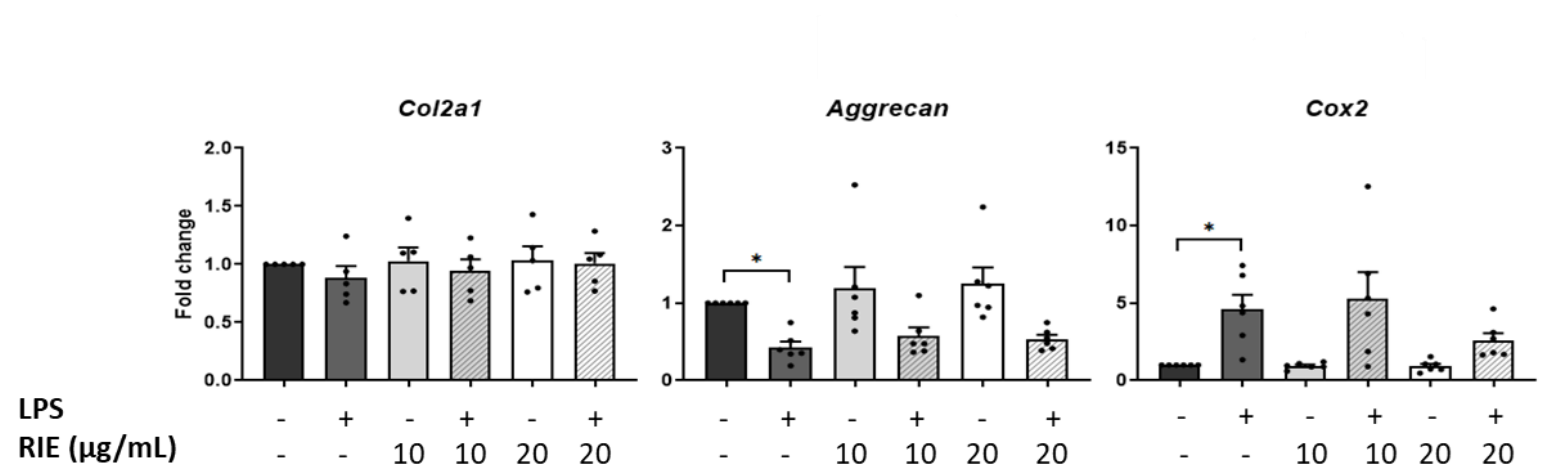

Figure 4. Effects in murine chondrocytes cultured with supernatant macrophage primed with Rubus idaeus extracts. (A) qPCR analysis of catabolic genes Mmp-3, Mmp-13, and Adamts-5, (B) anabolic genes Col2a1 and Aggrecan, and inflammatory gene Cox 2 in chondrocytes cultured with macrophages supernatant pre-treated $24 \mathrm{~h}$ with RIE (10 or $20 \mu \mathrm{g} / \mathrm{mL})$, then treated for $2 \mathrm{~h}$ with LPS $(100 \mathrm{ng} / \mathrm{mL})$, washed, and cultured for $24 \mathrm{~h}$ in fresh media. Data are mean \pm SEM from six experiments. ${ }^{*} p<0.05$.

To assess the expression at the protein level, the expression of MMP-3 and COX was measured by Western blot in lysates of chondrocytes cultured with RIE and/or LPS (Figure 5). Compared to controls, the MMP-3 expression was similar in chondrocytes cultured with the secretome of RIE-primed macrophages at the 2 doses. However, the MMP-3 expression decreased in the chondrocytes cultured with secretome of RIE-primed macrophages and then stimulated with LPS compared to LPS alone (10.16 \pm 3.43 -fold with LPS to $5.93 \pm 2.06$-fold and $4.70 \pm 0.79$-fold with 10 and $20 \mu \mathrm{g} / \mathrm{mL}$ of RIE, respectively) (Figure 5), although not significantly. Compared to controls, COX expression was lower in chondrocytes cultured with the secretome of RIE-primed macrophages at the two doses and stimulated with LPS (6.78 \pm 2.06 -fold with LPS to $3.03 \pm 0.69$-fold and $3.43 \pm 0.45$-fold with 10 and $20 \mu \mathrm{g} / \mathrm{mL}$ of RIE) (Figure 5). Compared to the secretome of LPS-activated macrophages alone, the secretome of RIE-primed macrophages reduced the expression of Cox2 in chondrocytes, although not significantly. 

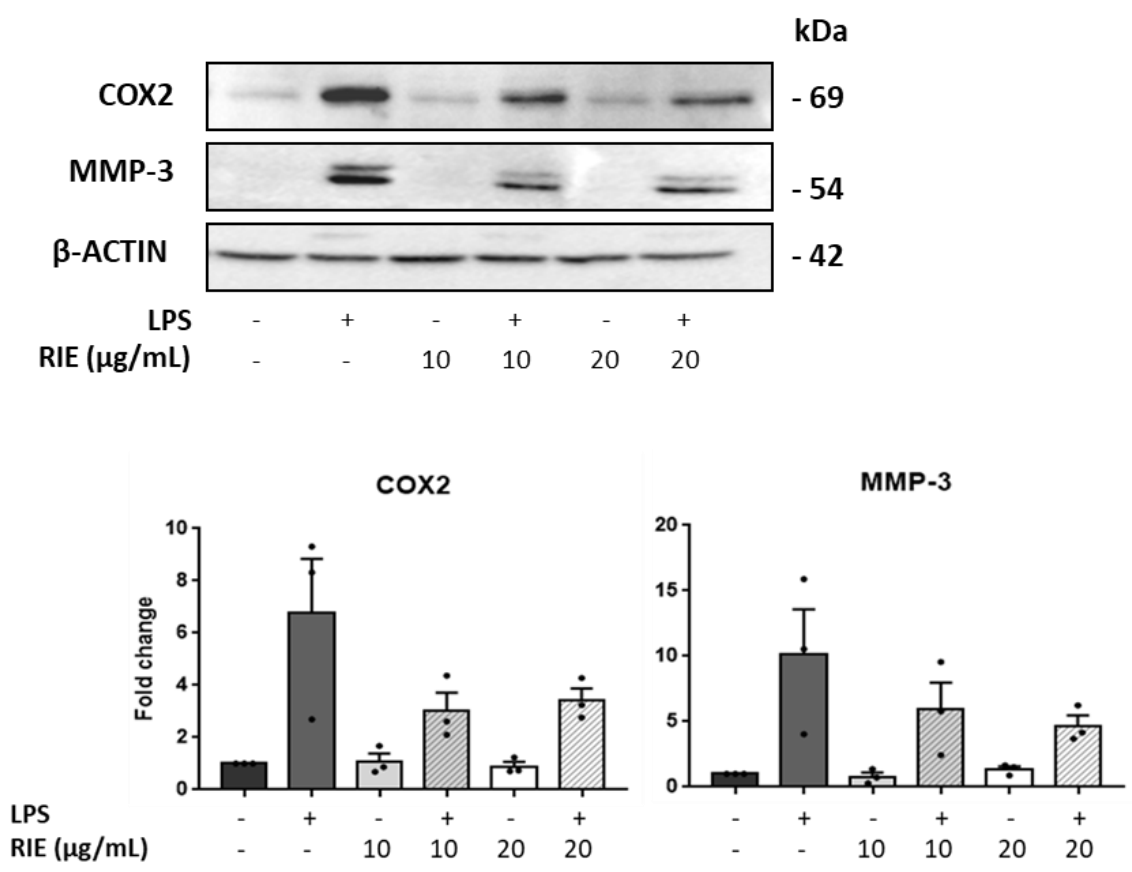

Figure 5. Effects of secretome of RIE-treated macrophages in Cox 2 and MMP-3 protein levels in murine chondrocytes. Western blot analysis and quantification of Cox 2 and matrix metalloproteinases MMP-3 in protein lysate of chondrocytes cultured with the supernatant of macrophages pre-treated $24 \mathrm{~h}$ with RIE (10 or $20 \mu \mathrm{g} / \mathrm{mL})$, then stimulated for $2 \mathrm{~h}$ with LPS $(100 \mathrm{ng} / \mathrm{mL})$, washed, and cultured for $24 \mathrm{~h}$ in fresh media. Data are mean \pm SEM from three experiments.

\section{Discussion}

In the present study, RIE tend to decrease cartilage loss and to reduce chondrocyte catabolism through a reduction in $I l 1$ and $I l 6$ expression by macrophages. Assessing first the direct effect of RIE in chondrocytes, we found activation in catabolic genes and an inhibition in anabolic genes that could suggest disequilibrium of the balance leading to cartilage loss. Although no direct effect has been reported in chondrocytes, this was an unexpected result as RIE has been reported to reduce deleterious cell function such as oxidative stress and NO synthesis in macrophages and skin cells [21]. Here, we found that RIE reduced Col10 expression, indicating that the major mechanisms are chondrocytes catabolism and hypertrophy. The latter is a main mechanism of cartilage degeneration that could compensate for the disrupted metabolic activity in chondrocytes. However, compared to chondrocytes primed with Il1, RIE worsened both catabolic and anabolic effects, which is not what is expected for an anti-osteoarthritic drug. Cartilage loss observed in OA involves the regulation of chondrocyte function that is under the influence of interactions between cartilage matrix and chondrocytes and diffusible factors released from synovial tissues and subchondral bone [21-23]. To better delineate the effects at the tissue level, we tested RIE in murine cartilage explants and showed that RIE prevented the degradation of sulfated proteoglycan as revealed by Safranin O staining. These data are in line with the reduction in proteoglycan release from bovine cartilage explants in which RIE altered the collagenolytic activity via a downregulation of MMP-2 and MMP-9. This was further confirmed in A549 cancer cells [24]. These data suggest that the protection in cartilage damage is partly mediated by MMPs that preserve the breakdown of the extracellular matrix. However, subchondral bone could have contributed to the prevention of proteoglycan loss. Although no effect of RIE has been described in bone cells, RIE could have modified the release of MMP or inflammatory cytokines by bone cells or bone marrow cells that, in turn, could prevent NITEGE neoepitope expression or cartilage loss. Nevertheless, RIE alone did not protect from the catabolic nor the anabolic pathway, but RIE conversely promoted catabolic signaling, which does not make it suitable for an 
OA drug. Further experiments using animal models for the development of OA in mice are awaited.

Red raspberry extracts possess anti-inflammatory properties as shown by the reduction in joint lesions in antigen-induced arthritis mice [17]. Degraded matrix products promote the release of several cytokines and other inflammatory factors by the synovial cells that, in turn, induced chondrocyte catabolism [1]. Therefore, we speculated that the low-grade inflammation associated with cartilage damage could be dampened by RIE in OA. Here, there was a trend towards of a reduction in LPS-induced Il1 and Il6 expression by RIE that contribute to reduce the inflammatory response of the macrophage. The lack of significance may be due to the lack of statistical power. Of note, RIE failed to inhibit the expression of Cox2 as well as Tnf expression as previously demonstrated in skin cells. This might be related to various inflammatory responses according to cell types. Because synovial inflammation plays an important role in cartilage breakdown, we hypothesized that macrophage secretome primed by RIE could modify the chondrocyte function. Hence, secretome of RIE-primed macrophages dose dependently decreased the expression of $\mathrm{Mmp}$ 3, Mmp-13, and Adamts-5 without reaching significance. This indicates that the inhibition of expression of matrix proteases is also driven by cytokine concentration derived from the joint tissue environment. Interestingly, the impact of RIE was restricted to catabolic processes since Col2A1 or Aggrecan anabolic genes were not modulated. This is contrasting to the upregulation of type 1 collagen synthesis in skin fibroblasts [25] driven by the MAPK pathway, which, again, illustrates a cell-dependent effect of RIE. However, experiments in explants showed that RIE alone prevented cartilage loss at a high dose and the secretome of RIE-primed macrophages also reduced the protein expression of MMP-3 and Cox-2. Although not identified, several factors produced upon RIE stimulation such as PGE2 or ROS could inhibit protease expression through the MAP kinase pathway [26] or an epigenetic regulation of Cox 2 as observed in fibroblasts [27]. In addition, the main effects are shown at the protein levels, suggesting that secretome could induce post translational modifications of $\operatorname{Cox} 2$ that contribute to reduce chondrocyte activity.

Altogether, this in vitro study provides insights that RIE tend to reduce the content of the cartilage matrix as well as a trend towards a reduction in inflammation response in order to prevent the release of metalloproteinases. Therefore, further experiments are required to show that RIE could have an effect in cartilage breakdown. More studies are needed to investigate the effect of RIE for the prevention of osteoarthritis.

Supplementary Materials: The following are available online at https:/ /www.mdpi.com/2218-273 X/11/2/245/s1, Figure S1: Cell viability of macrophages does not change with treatment of LPS or RIE.

Author Contributions: M.B.: performed and analyzed the experiments, wrote the manuscript. M.Z:: performed and analyzed the experiments. R.L.C.: conceptualization and reviewed the manuscript. P.F.-B.: designed the experiments and reviewed the manuscript. E.H.: designed and supervised the experiment, reviewed the manuscript. M.C.-S.: designed, supervised the experiments and wrote the manuscript. All authors have read and agreed to the published version of the manuscript.

Funding: This study was funded by Naturex, part of Givaudan.

Data Availability Statement: Data of the study will be available upon request after agreement of the authors.

Conflicts of Interest: The work was supported by a funding provided by Naturex SA, part of Givaudan.

$\begin{array}{ll}\text { Abbreviations } \\ \text { DMEM } & \text { Dulbecco's Modified Eagle Medium } \\ \text { FCS } & \text { Fetal Calf Serum } \\ \text { GAPDH } & \text { Glyceraldehyde-3-Phosphate Dehydrogenase } \\ \text { IL1 } & \text { Interleukin 1 } \\ \text { LPS } & \text { Lipopolysaccharides }\end{array}$




$\begin{array}{ll}\text { OA } & \text { Osteoarthritis } \\ \text { OCT } & \text { Optimal Cutting Temperature } \\ \text { qPCR } & \text { quantitative Polymerase Chain Reaction } \\ \text { RIE } & \text { Rubus idaeus extracts } \\ \text { TBP } & \text { TATA-Box Binding Protein }\end{array}$

\section{References}

1. Hunter, D.J.; Bierma-Zeinstra, S. Osteoarthritis. Lancet 2019, 393, 1745-1759. [CrossRef]

2. O'Neill, T.W.; McCabe, P.S.; McBeth, J. Update on the epidemiology, risk factors and disease outcomes of osteoarthritis. Best Pract. Res. Clin. Rheumatol. 2018, 32, 312-326. [CrossRef] [PubMed]

3. de Lange-Brokaar, B.J.; Ioan-Facsinay, A.; van Osch, G.J.; Zuurmond, A.M.; Schoones, J.; Toes, R.E.; Huizinga, T.W.; Kloppenburg, M. Synovial inflammation, immune cells and their cytokines in osteoarthritis: A review. Osteoarthr. Cartil. 2012, 20, 1484-1499. [CrossRef]

4. Griffin, T.M.; Scanzello, C.R. Innate inflammation and synovial macrophages in osteoarthritis pathophysiology. Clin. Exp. Rheumatol. 2019, 37 (Suppl. 120), 57-63.

5. Fahy, N.; de Vries-van Melle, M.L.; Lehmann, J.; Wei, W.; Grotenhuis, N.; Farrell, E.; van der Kraan, P.M.; Murphy, J.M.; Bastiaansen-Jenniskens, Y.M.; van Osch, G.J. Human osteoarthritic synovium impacts chondrogenic differentiation of mesenchymal stem cells via macrophage polarisation state. Osteoarthr. Cartil. 2014, 22, 1167-1175. [CrossRef]

6. Fernandes, T.L.; Gomoll, A.H.; Lattermann, C.; Hernandez, A.J.; Bueno, D.F.; Amano, M.T. Macrophage: A Potential Target on Cartilage Regeneration. Front. Immunol. 2020, 11, 111. [CrossRef]

7. Jayakumar, T.; Saravana Bhavan, P.; Sheu, J.R. Molecular Targets of Natural Products for Chondroprotection in Destructive Joint Diseases. Int. J. Mol. Sci. 2020, 21, 4931. [CrossRef]

8. Henrotin, Y.; Mobasheri, A. Natural Products for Promoting Joint Health and Managing Osteoarthritis. Curr. Rheumatol. Rep. 2018, 20, 72. [CrossRef]

9. Pagani, S.; Minguzzi, M.; Sicuro, L.; Veronesi, F.; Santi, S.; Scotto D'Abusco, A.; Fini, M.; Borzì, R.M. The N-Acetyl Phenylalanine Glucosamine Derivative Attenuates the Inflammatory/Catabolic Environment in a Chondrocyte-Synoviocyte Co-Culture System. Sci. Rep. 2019, 9, 13603. [CrossRef]

10. Gregori, D.; Giacovelli, G.; Minto, C.; Barbetta, B.; Gualtieri, F.; Azzolina, D.; Vaghi, P.; Rovati, L.C. Association of Pharmacological Treatments With Long-term Pain Control in Patients With Knee Osteoarthritis: A Systematic Review and Meta-analysis. JAMA 2018, 320, 2564-2579. [CrossRef]

11. Lomonte, A.B.V.; Mendonça, J.A.; de Brandão, G.C.; Castro, M.L. Multicenter, randomized, double-blind clinical trial to evaluate efficacy and safety of combined glucosamine sulfate and chondroitin sulfate capsules for treating knee osteoarthritis. Adv. Rheumatol. 2018, 58, 41. [CrossRef]

12. Diaconeasa, Z.; Iuhas, C.I.; Ayvaz, H.; Rugină, D.; Stanilă, A.; Dulf, F.; Bunea, A.; Socaci, S.A.; Socaciu, C.; Pintea, A. Phytochemical Characterization of Commercial Processed Blueberry, Blackberry, Blackcurrant, Cranberry, and Raspberry and Their Antioxidant Activity. Antioxidants 2019, 8, 540. [CrossRef]

13. Kim, H.R.; Jeong, D.H.; Kim, S.; Lee, S.W.; Sin, H.S.; Yu, K.Y.; Jeong, S.I.; Kim, S.Y. Fermentation of Blackberry with L. plantarum JBMI F5 Enhance the Protection Effect on UVB-Mediated Photoaging in Human Foreskin Fibroblast and Hairless Mice through Regulation of MAPK/NF-кB Signaling. Nutrients 2019, 11, 2429. [CrossRef]

14. Chaves, V.C.; Soares, M.S.P.; Spohr, L.; Teixeira, F.; Vieira, A.; Constantino, L.S.; Pizzol, F.D.; Lencina, C.L.; Spanevello, R.M.; Freitas, M.P.; et al. Blackberry extract improves behavioral and neurochemical dysfunctions in a ketamine-induced rat model of mania. Neurosci. Lett. 2020, 714, 134566. [CrossRef]

15. Tu, L.; Sun, H.; Tang, M.; Zhao, J.; Zhang, Z.; Sun, X.; He, S. Red raspberry extract (Rubus idaeus L shrub) intake ameliorates hyperlipidemia in HFD-induced mice through PPAR signaling pathway. Food Chem. Toxicol. 2019, 133, 110796. [CrossRef]

16. Ghalayini, I.F.; Al-Ghazo, M.A.; Harfeil, M.N. Prophylaxis and therapeutic effects of raspberry (Rubus idaeus) on renal stone formation in Balb/c mice. Int. Braz J. Urol 2011, 37, 259-266. [CrossRef] [PubMed]

17. Jean-Gilles, D.; Li, L.; Ma, H.; Yuan, T.; Chichester, C.O., 3rd; Seeram, N.P. Anti-inflammatory effects of polyphenolic-enriched red raspberry extract in an antigen-induced arthritis rat model. J. Agric. Food Chem. 2012, 60, 5755-5762. [CrossRef]

18. Singleton, V.L.; Rossi, J.A. Colorimetry of total phenolics with phosphomolybdic-phosphotungstic acid reagents. Am. J. Enol. Vitic. 1965, 16, 144-158.

19. Bouaziz, W.; Funck-Brentano, T.; Lin, H.; Marty, C.; Ea, H.K.; Hay, E.; Cohen-Solal, M. Loss of sclerostin promotes osteoarthritis in mice via $\beta$-catenin-dependent and -independent Wnt pathways. Arthritis Res Ther. 2015, 17, 24. [CrossRef]

20. Funck-Brentano, T.; Bouaziz, W.; Marty, C.; Geoffroy, V.; Hay, E.; Cohen-Solal, M. Dkk-1-mediated inhibition of Wnt signaling in bone ameliorates osteoarthritis in mice. Arthritis Rheumatol. 2014, 66, 3028-3039. [CrossRef]

21. van de Velde, F.; Esposito, D.; Grace, M.H.; Pirovani, M.E.; Lila, M.A. Anti-inflammatory and wound healing properties of polyphenolic extracts from strawberry and blackberry fruits. Food Res. Int. 2019, 121, 453-462. [CrossRef]

22. Chevalier, X.; Eymard, F.; Richette, P. Biologic agents in osteoarthritis: Hopes and disappointments. Nat. Rev. Rheumatol. 2013, 9, 400-410. [CrossRef] 
23. Funck-Brentano, T.; Cohen-Solal, M. Crosstalk between cartilage and bone: When bone cytokines matter. Cytokine Growth Factor Rev. 2011, 22, 91-97. [CrossRef]

24. Hsieh, Y.S.; Chu, S.C.; Hsu, L.S.; Chen, K.S.; Lai, M.T.; Yeh, C.H.; Chen, P.N. Rubus idaeus L. reverses epithelial-to-mesenchymal transition and suppresses cell invasion and protease activities by targeting ERK1/2 and FAK pathways in human lung cancer cells. Food Chem. Toxicol. 2013, 62, 908-918. [CrossRef]

25. Gao, W.; Wang, Y.S.; Hwang, E.; Lin, P.; Bae, J.; Seo, S.A.; Yan, Z.; Yi, T.H. Rubus idaeus L. (red raspberry) blocks UVB-induced MMP production and promotes type I procollagen synthesis via inhibition of MAPK/AP-1, NF- $\beta \beta$ and stimulation of TGF- $\beta /$ Smad, Nrf2 in normal human dermal fibroblasts. J. Photochem. Photobiol. B 2018, 185, 241-253. [CrossRef]

26. Yoon, H.Y.; Lee, E.G.; Lee, H.; Cho, I.J.; Choi, Y.J.; Sung, M.S.; Yoo, H.G.; Yoo, W.H. Kaempferol inhibits IL-1 $\beta$-induced proliferation of rheumatoid arthritis synovial fibroblasts and the production of COX-2, PGE2 and MMPs. Int. J. Mol. Med. 2013, 32, 971-977. [CrossRef]

27. Cao, K.; Wei, L.; Zhang, Z.; Guo, L.; Zhang, C.; Li, Y.; Sun, C.; Sun, X.; Wang, S.; Li, P.; et al. Decreased histone deacetylase 4 is associated with human osteoarthritis cartilage degeneration by releasing histone deacetylase 4 inhibition of runt-related transcription factor-2 and increasing osteoarthritis-related genes: A novel mechanism of human osteoarthritis cartilage degeneration. Arthritis Res. Ther. 2014, 16, 491. 